\title{
SCIENTIFIC APPLICATIONS IN THE CLOUD: RESOURCE OPTIMISATION BASED ON METAHEURISTICS
}

\author{
ANAS MOKHTARI, MOSTAFA AZIZI ${ }^{\dagger}$ AND MOHAMMED GABLI ${ }^{\ddagger}$
}

\begin{abstract}
The advent of emerging technologies such as 5G and Internet of Things (IoT) will generate a colossal amount of data that should be processed by the cloud computing. Thereby, cloud resources optimisation represents significant benefits in different levels: cost reduction for the user, saving energy consumed by cloud data centres, etc. Cloud resource optimisation is a very complex task due to its NP-hard characteristic. In this case, use of metaheuristic approaches is more rational. But the quality of metaheuristic solutions changes by changing the problem. In this paper we have dealt with the problem of determining the configuration of resources in order to minimise the payment cost and the duration of the scientific applications execution. For that, we proposed a mathematical model and three metaheuristic approaches, namely the Genetic Algorithm (GA), hybridisation of the Genetic Algorithm with Local Search (GA-LS) and the Simulated Annealing (SA). The comparison between them showed that the simulated annealing finds more optimal solutions than those proposed by the genetic algorithm and the GA-LS hybridisation.
\end{abstract}

Key words: Cloud computing, Resources Management, Optimisation, Metaheuristic, Artificial Intelligence

AMS subject classifications. 68M14, 68T01, 90C10

1. Introduction. The cloud computing is a distributed computer system based on an emergent technologies like the virtualisation. By comparing it with the conventional distributed computing, the latter has the objective of providing a collaborative sharing of resources to which users are linked, while the cloud computing has the objective of providing services or applications with ensuring of the scaling, the transparency (vis-a-vis the physical implementation of the cloud), the security, the supervision and the management.

There are three main types of cloud models:

IaaS (Infrastructure as a Service) offers a low level computing resources in the form of Virtual Machines (VM). EC2 [1] and Azure [3] are two IaaS examples provided respectively by Amazon and Microsoft.

PaaS (Platform as a Service) offers ready to use software platforms on which users develop and deploy their applications. Heroku [6] and Google App Engine [4] provide this type of cloud.

SaaS (Service as a Service) offers ready-to-use applications. It's the highest level in the cloud. G Suite [5] (Google) and Office 365 [7] (Microsoft) are SaaS models.

There are numerous applications that require High Performance Computing (HPC). Weather forecast, chemical process modeling, and the physics simulations are examples of such applications. Since the available computing resources in the cloud are very efficient, users of the HPC applications showed interest in running their intensive applications in the cloud environment.

Cloud computing face several challenges and problems that are subject to scientific research. The excessive consumption of the electric energy and the performance degradation of the application execution because of underestimation of reserved resources are examples of problems that cloud systems face.

This work addresses the problem of performance and cost of applications execution in the cloud. It's a double contradictory objectives problem. The first one is to maximise the resources to be used to have a good performance by reducing the execution time. The second one is to minimise the resources to be used to reduce the payment cost.

There are several works in the literature which have dealt with this type of problem. The complexity of the cloud resource optimisation problem has led researchers to use metaheuristics. For instance, in our

*MATSI Lab., ESTO, University Mohammed I ${ }^{\text {st }}$, Oujda, Morocco (a.mokhtari@ump.ac.ma).

${ }^{\dagger}$ MATSI Lab., ESTO, University Mohammed Ist, Oujda, Morocco (azizi.mos@ump.ac.ma).

${ }^{\ddagger}$ LARI Lab., FSO, University Mohammed I ${ }^{\text {st }}$, Oujda, Morocco (medgabli@ump.ac.ma). 


\begin{tabular}{cl}
\hline Variable & Definition \\
\hline$P$ & Set of packages types offered by a cloud provider during a set of time periods \\
$C_{M}$ & Set of consumer requirements as the maximum cost \\
$T_{M}$ & Maximum time for execution \\
$D_{S}$ & Disk storage \\
$M_{C}$ & Memory capacity \\
$G_{f}$ & Processing demand in Gflop \\
$p$ & A package type from the set $P(p \in P)$ \\
$c_{p}$ & The cost of purchasing the package $p$ for one period of time \\
$d_{p}$ & Disk storage computing resource for the package $p$ \\
$m_{p}$ & Memory capacity for the package $p$ \\
$g_{p}$ & Processing power for the package $p$ \\
$N_{M}$ & Maximum limit of packages that a consumer can purchase at a period of time \\
\hline
\end{tabular}

previous works $[20,21,22]$, we considered weighted objective functions in our model and chose the approach of genetic algorithms for dealing with this issue. The authors of [25] investigated the meta-heuristic resource allocation techniques used in the IaaS cloud computing environment. Jena et al. [26] proposed a hybridisation of modified Particle swarm optimization (MPSO) and improved Q-learning algorithm to load balancing of tasks on the cloud environment. Coutinho et al. [10] implemented an ILP model and then used Greedy Randomised Adaptive Search Procedure (GRASP) to solve the resource optimisation problem. Except that the proposed metaheuristics has been compared with a deterministic algorithm. Due to difficulty of this problem, which is classified as NP-hard [10], most of these works have tried to solve it using metaheuristic approaches. The effectiveness of these approaches in terms of the solutions found varies from one problem to another.

In this work, we used three metaheuristic algorithms: Genetic Algorithm (GA), Simulated Annealing (SA) and hybridisation of GA and Local Search method (GA-LS). Our goal is executing demanding HPC applications in the IaaS cloud.

The remaining of this paper is organised as follows: in the Sect. 2, we describe the mathematical model used. In the Sect. 3, we explain the three proposed resolution metaheuristics. In the Sect. 4, We compare between these approaches by experiments, then analyse the obtained results. We conclude in Sect. 5 .

2. Mathematical model. In the infrastructure cloud (IaaS), resources are the virtual CPUs (vCPU), the memory, the storage drives, etc. of the VMs. Each resource type has the same characteristics as the equivalent physical resources (like processor frequency for the vCPU).

Cloud infrastructure service providers make available to the users a variety of VM choices. Each type has a specific computing power and usage price per hour.

The mathematical model that we present is based on the variables cited in the Table 2.1.

Our optimisation problem is composed of two objective functions: (i) minimise the total cost and (ii) minimise the execution time. We transformed them to a unique objective function by their sum with coefficients assigned to each of these two functions (weighted sum). So, we have [10]:

$$
(\mathrm{CC} \text { ILP }) \quad \min \left(\alpha f_{1}+(1-\alpha) f_{2}\right)
$$

with

$$
f_{1}=\sum_{p \in P} \sum_{i=1}^{N_{M}} \sum_{t \in T} c_{p} x_{p i t}
$$

and

$$
f_{2}=t_{m}
$$


subject to

$$
\begin{aligned}
& \sum_{p \in P} \sum_{i=1}^{N_{M}} \sum_{t \in T} c_{p} x_{p i t} \leq C_{M} \\
& \sum_{p \in P} \sum_{i=1}^{N_{M}} d_{p} x_{p i t} \geq D_{s} x_{p^{\prime} i^{\prime} t}, \quad \forall t \in T, \forall p^{\prime} \in P, \forall i^{\prime} \in\left\{1, \ldots, N_{M}\right\} \\
& \sum_{p \in P} \sum_{i=1}^{N_{M}} m_{p} x_{p i t} \geq M_{C} x_{p^{\prime} i^{\prime} t}, \quad \forall t \in T, \forall p^{\prime} \in P, \forall i^{\prime} \in\left\{1, \ldots, N_{M}\right\} \\
& \sum_{p \in P} \sum_{i=1}^{N_{M}} \sum_{t \in T} g_{p} x_{p i t} \geq G_{f} \\
& \sum_{p \in P} \sum_{i=1}^{N_{M}} x_{p i t} \leq N_{M}, \quad \forall t \in T \\
& t_{m} \geq t x_{p i t}, \quad \forall t \in T, \forall p \in P, \forall i \in\left\{1, \ldots, N_{M}\right\} \\
& x_{p i t+1} \leq x_{p i t}, \quad \forall t \in T, \forall p \in P, \forall i \in\left\{1, \ldots, N_{M}\right\} \\
& x_{p i+1 t} \leq x_{p i t}, \quad \forall t \in T, \forall p \in P, \forall i \in\left\{1, \ldots, N_{M}-1\right\} \\
& x_{p i t} \in\{0,1\}, \quad \forall t \in T, \forall p \in P, \forall i \in\left\{1, \ldots, N_{M}\right\} \\
& t_{m} \in \mathbb{Z}
\end{aligned}
$$

The terms $\alpha$ and $(1-\alpha)$ represent the weights of the two objectives. To automate the choice of the weights and ensure a fair treatment between the two objective functions [12], we used dynamic weights to meet the condition (2.14):

$$
\left|\alpha(t) f_{1}-(1-\alpha(t)) f_{2}\right| \prec \varepsilon,
$$

where $\varepsilon$ is a positive number in the vicinity of $0, t$ is a time-step, and $\alpha(t)$ and $(1-\alpha(t))$ are the dynamic weights. $\alpha(t)$ is calculated by the formula (2.15):

$$
\alpha(t)=\frac{\left|f_{2}\left(x_{t-1}\right)\right|}{\left|f_{1}\left(x_{t-1}\right)\right|+\left|f_{2}\left(x_{t-1}\right)\right|}
$$

where $x_{t-1}$ is the best solution of the iteration $(t-1)$ of the metaheuristic. For more details, see our previous work [20]. 


\begin{tabular}{cl}
\hline Variable & Definition \\
\hline$S$ & Solution that represents a combination of packages. \\
$\Delta$ & The difference between the value of the optimisation function of the \\
$T$ & current solution and that under the evaluation. \\
\hline
\end{tabular}

\section{Metaheuristic resolution approaches.}

3.1. Simulated Annealing. The origin of this method come from the experiments done by Metropolis et al. [19] to simulate the stochastic evolution of such physical system. In the context of minimisation of the objective function $f$, the process of simulated annealing is illustrated in the Algorithm 5. Table 3.1 defines the used variables in this algorithm.

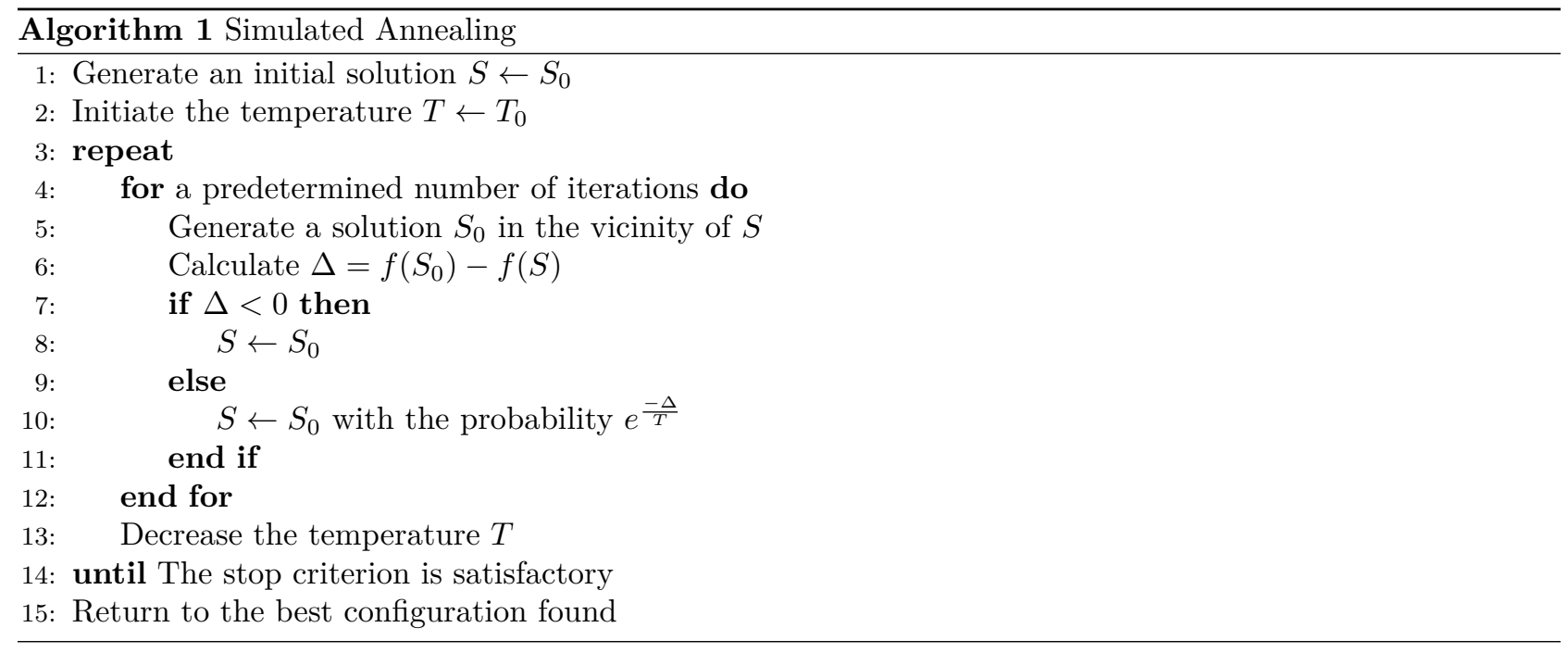

The proper functioning of the Simulated Annealing algorithm depends on the configuration space and the temperature decrease function. For example, Dréo et al. [11] and Kirkpatrick et al. [18] propose methods to define this function.

3.2. Genetic Algorithm. The Genetic Algorithm is an optimisation technique that mimic the natural evolution. The first work on the GA was developed by John Holland in 1962 [16]. The popularity of the GA returns to the work of David Golberg [14]. In this context, we call chromosome $x$ any proposed solution by the GA. This chromosome is composed of a set of values called genes. The Algorithm 6 presents the second approach. Table 3.2 describes used variables.

The fitness function permits to evaluate each chromosome by assigning it a value that depends on the quality of this solution (chromosome). The crossover operation randomly chooses two chromosomes and a position of crossing. Then, we exchange the bits which are very close to the position of crossing. The mutation operation is to select a gene and replace its value by another randomly chosen from a given set.

3.3. Hybridisation of GA and Local Search method. The metaheuristic methods of optimisation are classified into two categories: approaches which aim at diversification and those which aim at intensification in the research space. The main difference between these two categories [13] is that in intensification, research focuses on the evaluation of neighbours of elite solutions, while diversification encourages the research process to evaluate regions not visited and to generate solutions that differ significantly from the solutions seen previously. 


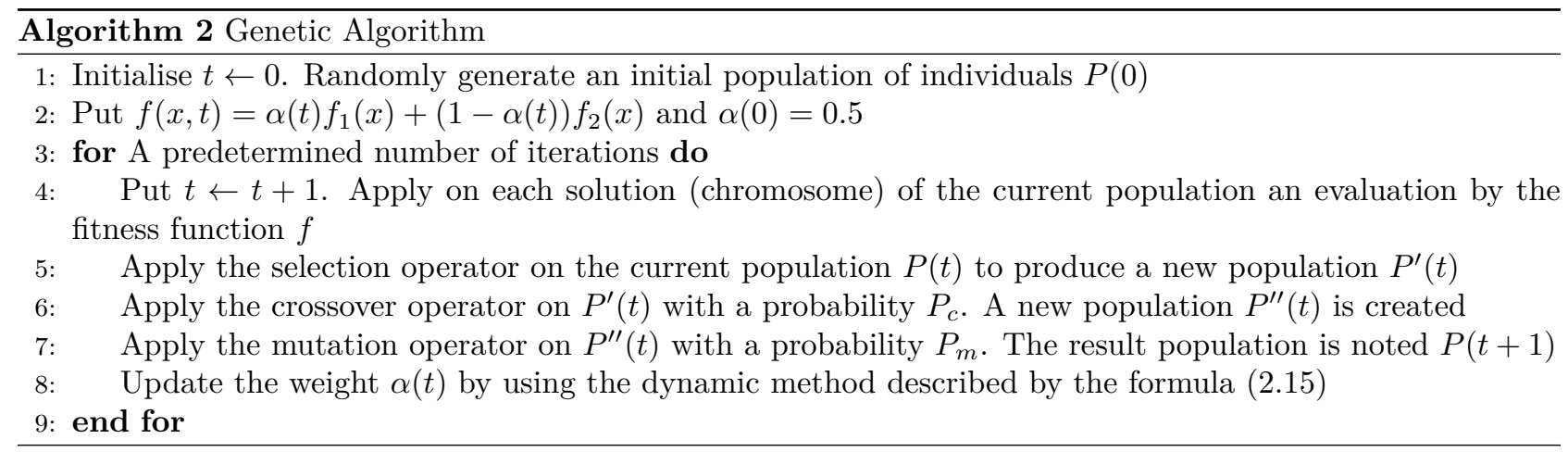

TABLE 3.2

Definitions of the genetic algorithm variables

\begin{tabular}{cl}
\hline Variable & Definition \\
\hline$P(t)$ & Population composed of several candidate solutions used in the iteration $t$. \\
$x$ & A solution that represents a combination of packages. \\
$P_{c}$ & Crossover probability. \\
$P_{m}$ & Mutation probability. \\
\hline
\end{tabular}

GA are based on diversification, while the Local Search (LS) algorithm intensifies its search in the vicinity of a solution.

Evolutionary algorithms, including GA, suffer from the inability to intensify research enough. As a result, they cannot effectively achieve high quality candidate solutions [17]. A significant improvement in the performance of the GA for combinatorial problems can be ensured by the application of LS on the solution found by the GA. We talk about hybridisation between GA and LS (GA-LS).

The third approach is presented by the Algorithm 7. We are considering the improvement of the solution proposed by the GA using LS as a method of intensification [8]. The first part of this algorithm (lines 1 to 10) represents the GA. It consists in initialising a population of candidate solutions, then repetitively carrying out the operations of evaluation, selection, crossing and mutation. In the second part (lines 11 to 18), which represents the LS algorithm, the best solution found by the GA, noted $s$, is selected to become the initial configuration of LS.

\section{Comparison of the Results.}

4.1. Description of data. One of the most important parts of a comparison between metaheuristics is the testbed on which it is made [23]. It is preferable to develop a new test bed (program) to implement the metaheuristics and compare between them. In addition, so that the execution time of each algorithm is comparable to the others, the best approach to use consists in implementing these algorithms by the same programming language, compiling them in the same machine with the same parameters (flags) of compilation and run them in the same machine [23].

We implemented our approaches in Java language, then compiled the code by the javac compiler (OpenJDK), version 11.0.6, on a machine characterised by an Intel processor ${ }^{\circledR}$ Core $^{\mathrm{TM}} \mathrm{i} 7-2670 \mathrm{QM}$ which contains eight cores clocked at $2.20 \mathrm{GHz}$. We tested our programs on the same computer using the Java virtual machine (OpenJDK JRE), version 11.0.6.

We have applied these algorithms on four instances of applications with different sizes: an application which deals with the problem of manipulation of the biological sequence $(\mathrm{raxml})$ [24], a typical analysis application for the CMS experience (CMS-1500) [9] and two applications for solving the $\mathrm{QAP}^{1}$ problem by the separation and

\footnotetext{
${ }^{1}$ Quadratic Assignment Problem
} 


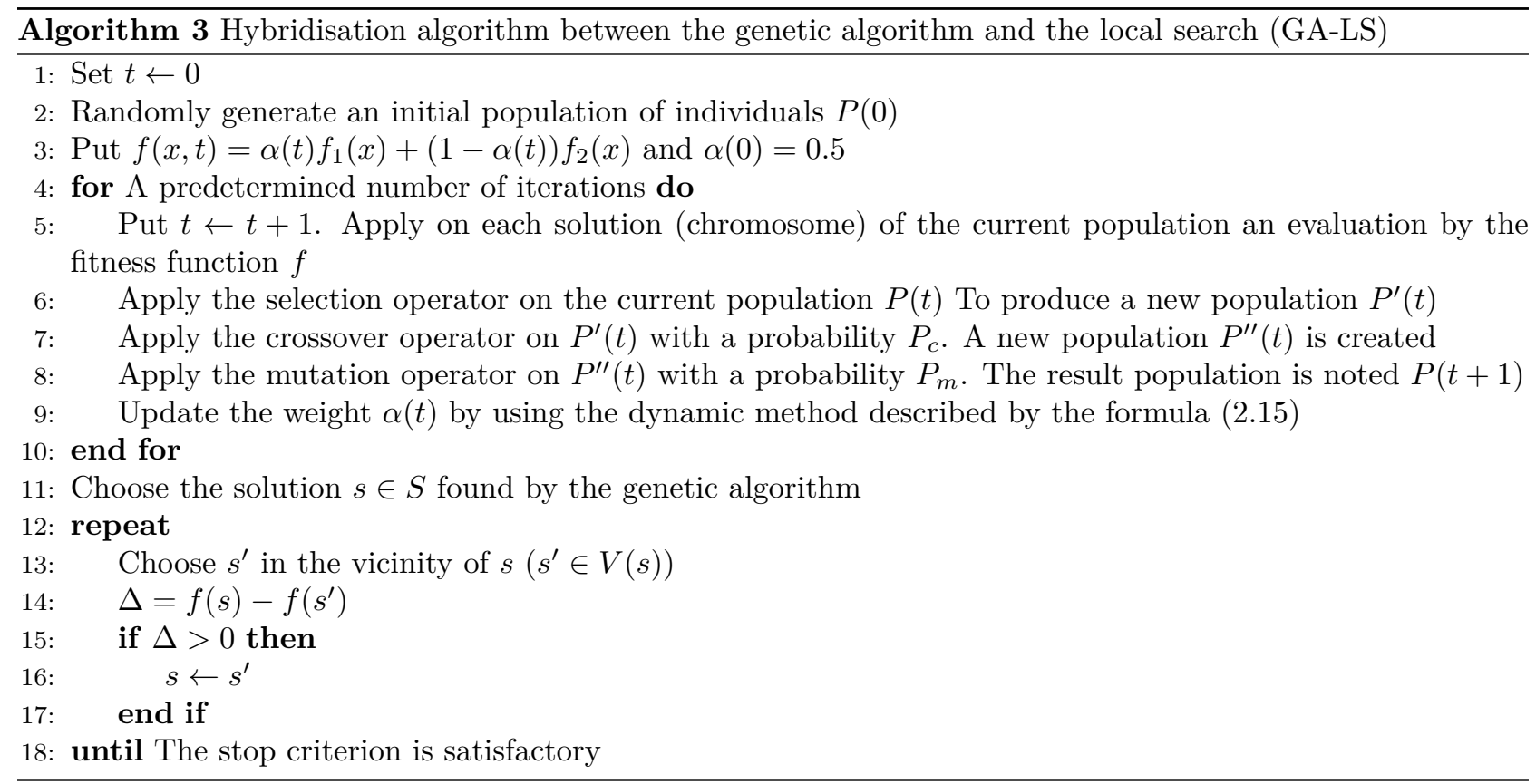

TABLE 4.1

Characteristics of the applications used in the experiment [10]

\begin{tabular}{ccccccc}
\hline Application & $\begin{array}{c}\text { Memory } \\
\text { (GB) }\end{array}$ & $\begin{array}{c}\text { Storage } \\
\text { (GB) }\end{array}$ & GFLOP & $\begin{array}{c}\text { Time } \\
\text { (hour) }\end{array}$ & $\begin{array}{c}\text { Max } \\
\text { packages }\end{array}$ & $\begin{array}{c}\text { Cost } \\
(\$)\end{array}$ \\
\hline raxml & 3 & 2 & $3,317,760$ & 10 & 20 & 50 \\
cms-1500 & 2,250 & 30 & $324,000,000$ & 10 & 20 & 75 \\
nug28-cbb & 528 & 528 & $541,765,325$ & 10 & 20 & 120 \\
nug30-cbb & 918 & 918 & $967,438,080$ & 15 & 30 & 250 \\
\hline
\end{tabular}

evaluation algorithm (nug28-cbb and nug30-cbb) [15]. The characteristics of these applications are summarised in the Table 4.1.

In order to have realistic results, we applied our simulation to the offers of two cloud computing providers: Amazon EC2 [1] and Google Compute Engine [2] (cf. Table 4.2).

For each application, we performed the metaheuristics several times to ensure the accuracy of the results obtained. Details are presented in Table 4.3.

To give metaheuristic approaches more chance of finding good solutions, we have extended the execution time for larger application problems (between 1 and 20 seconds, cf. Table 4.3-left). In addition, in order to verify the influence of this duration on the quality of the results obtained, we launched other tests with a long execution time ( $c f$. Table 4.3-right).

Since the LS depends on the solution found after the execution of the GA, and in order to be able to compare between the results of the GA and GA-LS hybridisation approaches, we have allocated to the LS part an execution time equivalent to approximately $8 \%$ of the execution time of the GA alone:

$$
\operatorname{Duration}(\mathrm{GA}-\mathrm{LS}) \approx 1.08 \times \operatorname{duration}(\mathrm{GA}) \Rightarrow \operatorname{Duration}(\mathrm{GA}-\mathrm{LS}) \approx \operatorname{duration}(\mathrm{GA})
$$

Generally, the parameters values of each algorithm must be determined by its designer because their change influences the performance of metaheuristics [23]. For the case of our approaches, we fixed the parameters 
TABLE 4.2

Cloud Provider's VM Instance Pricing

\begin{tabular}{cccccc}
\hline $\begin{array}{c}\text { Cloud } \\
\text { provider }\end{array}$ & $\begin{array}{c}\text { Instance } \\
\text { type }\end{array}$ & vCPU & $\begin{array}{c}\text { Memory } \\
\text { (GB) }\end{array}$ & $\begin{array}{c}\text { Storage } \\
(\mathbf{G B})\end{array}$ & $\begin{array}{c}\text { Price } \\
(/ \text { hour })\end{array}$ \\
\hline Amazon EC2 & c4.large & 2 & 3.75 & 200 & $\$ 0.128$ \\
& c4.xlarge & 4 & 7.5 & 400 & $\$ 0.255$ \\
& c4.2xlarge & 8 & 15 & 800 & $\$ 0.509$ \\
& c4.4xlarge & 16 & 30 & 1,600 & $\$ 1.018$ \\
& c4.8xlarge & 36 & 60 & 2,500 & $\$ 1.938$ \\
\hline Google Compute & n1-highcpu-2 & 2 & 1.80 & 100 & $\$ 0.0764$ \\
Engine & n1-highcpu-4 & 4 & 3.60 & 200 & $\$ 0.1529$ \\
& n1-highcpu-8 & 8 & 7.20 & 400 & $\$ 0.3058$ \\
& n1-highcpu-16 & 16 & 14.40 & 800 & $\$ 0.6116$ \\
& n1-highcpu-32 & 32 & 28.80 & 1,600 & $\$ 1.2233$ \\
& n1-highcpu-64 & 64 & 57.60 & 2,500 & $\$ 2.4077$ \\
\hline
\end{tabular}

TABle 4.3

Parameters used for the execution of the three approaches: GA, GA-LS hybridisation, and SA: for a no long duration (left) and for a long duration (right)

\begin{tabular}{ccc}
\hline Application & $\begin{array}{c}\text { Execution } \\
\text { time (s) }\end{array}$ & $\begin{array}{c}\text { Number of } \\
\text { trials }\end{array}$ \\
\hline raxml & 1 & 10 \\
cms-1500 & 5 & 10 \\
nug28-cbb & 10 & 10 \\
nug30-cbb & 20 & 10 \\
\hline
\end{tabular}

\begin{tabular}{ccc}
\hline Application & $\begin{array}{c}\text { Execution } \\
\text { time (s) }\end{array}$ & $\begin{array}{c}\text { Number of } \\
\text { trials }\end{array}$ \\
\hline raxml & 180 & 2 \\
cms-1500 & 180 & 2 \\
nug28-cbb & 180 & 2 \\
nug30-cbb & 180 & 2 \\
\hline
\end{tabular}

according to the application concerned. The details are presented in Table 4.4 for GA and GA-LS, and Table 4.5 for SA.

4.2. Results analysis. One of the most important points in the comparison between metaheuristics is the solution quality. The measurement of this quality is relative and it depends on the treated application. For long-term planning, the acceptable difference between the found and the optimal solutions is smaller than that of short-term planning applications [23]. For that, we must compare between the solutions found by metaheuristics and not determine if they reach a threshold of solution quality [23].

To compare the solutions found by metaheuristics, we must base ourselves on a metric. In our case, we have the possibility of using one of the following two metrics [23]:

- Deviation from best-known solutions for a problem;

- Deviation between the algorithms being compared: This method has the advantage of making the comparison between the algorithms very explicit. However, these comparisons lack any sense of the actual error of solutions.

In our knowledge, there is no best-known solutions for the case of our problem. So, we used the second metric.

The results of our experiment are detailed in the Table 4.6. We have limited ourselves in this table to the best solution found in the ten trials carried out by application.

In the Application column, we put the name of the problem instance to be executed with the name of the cloud on which this execution is planned. For example, the first line (raxml am) concerns the execution of raxml instance in the Amazon EC2 (am) cloud, while for the fifth line (raxml_go), we are talking about the same application in the Google Compute Engine (go) cloud.

The details of the solution for each metaheuristic are represented in five columns: the payment cost $f_{1}$ 
TABLE 4.4

Parameters used for the GA

\begin{tabular}{cccc}
\hline $\begin{array}{c}\text { Problem } \\
\text { instance }\end{array}$ & $\begin{array}{c}\text { Population } \\
\text { size }\end{array}$ & $\begin{array}{c}\text { Crossover } \\
\text { probability }\left(P_{c}\right)\end{array}$ & $\begin{array}{c}\text { Mutation } \\
\text { probability }\left(P_{m}\right)\end{array}$ \\
\hline raxml & 40 & 0.5 & 0.01 \\
cms-1500 & 40 & 0.5 & 0.01 \\
nug28-cbb & 40 & 0.5 & 0.01 \\
nug30-cbb & 60 & 0.5 & 0.01 \\
\hline \multicolumn{4}{c}{ TABLe 4.5 } \\
\multicolumn{4}{c}{ Parameters used for the SA } \\
\hline Problem instance & Annealing rate $(\lambda)$ & Initial temperature \\
\hline raxml & 0.9 & 100 \\
cms-1500 & 0.9 & 100 \\
nug28-cbb & 0.9 & 100 \\
nug30-cbb & 0.9 & \\
\hline
\end{tabular}

(in dollars) of the found solution, the duration $f_{2}$ (in hours) necessary for the execution of the application, the value of the weighted objective function $f, f_{\text {avg }}$ to check the robustness of this solution by calculating the average of the values of $f$ for the ten trials carried out $\left(f_{\text {avg }}=\sum_{i=1}^{10} \frac{f_{\text {trial }}}{10}\right)$, and the fifth column contains the execution time of the metaheuristic (in seconds) which allowed to have this result.

From Fig. 4.1, we can easily notice that, for all the tested applications, the best minimisation of the objective function $f$ is ensured by the SA, followed by the GA-LS hybridisation algorithm. The optimal cost of $f$ found by the SA is $53.34 \%$ (nug30-cbb_go) to $92.3 \%$ (raxml_go) less than that found by the GA, and $29.27 \%$ (nug28-cbb_go) to $90.57 \%$ (raxml_go) less than the GA-LS.

The fact that the GA-LS optimises the objective function better than the GA is expected since the GA-LS diverts the weakness of the GA in terms of intensification.

Since the function $f$ is the result of the weighted sum of the two functions $f_{1}$ and $f_{2}\left(f=\alpha f_{1}+(1-\alpha) f_{2}\right)$, we also analysed and compared these two objectives for the three metaheuristic approaches. The histograms in Fig. 4.2 and Fig. 4.3 represent, respectively, the values of $f_{1}$ and $f_{2}$.

Simulated Annealing gives us, for all the tested applications, cheaper solutions $\left(f_{1}\right)$ and faster execution $\left(f_{2}\right)$ than those proposed by the GA and the GA-LS. Solutions of the GA-LS hybridisation algorithm are more expensive than those found by the GA (case of nug28-cbb_go and nug30-cbb_go in Fig. 4.2), but are always faster in execution ( $c f$. Fig. 4.3).

Since the aforementioned results represent the best solutions found among the ten trials that we carried out, this brings us back to verifying that these solutions do not represent particular cases (noise). The robustness of the GA is presented in Fig. 4.4. The red (dark) bar represents the value $f$ and the red+blue (dark+light) bar represents $f_{\text {avg }}$. The best values of $f$ are $20.5 \%$ to $46.35 \%$ smaller than the average $f_{\text {avg }}$ of the solutions found by the ten trials. Similarly, $f$ of the GA-LS ( $c f$. Fig. 4.5 ) is $20.6 \%$ to $49.79 \%$ lower than $f_{\text {avg }}$. This variation is more reduced in the case of the SA (cf Fig. 4.6) since it is between $0.01 \%$ and $29.72 \%$. The long-term execution of the three algorithms (180 seconds) did not considerably improve the quality of the found solutions (details in the Table 4.6). In this case too, the SA gives in all the tested cases more optimal solutions than those of the GA and the GA-LS.

5. Conclusion. In this paper, we treated the problem of multi-objective optimisation of the cloud computing resources, for the execution of the intensive computing applications. We took into account the decrease in the budget by reducing the allocation price of computing resources (first objective) and the increase in performance by minimising the execution time (second objective). Then, we implemented three problem solving 


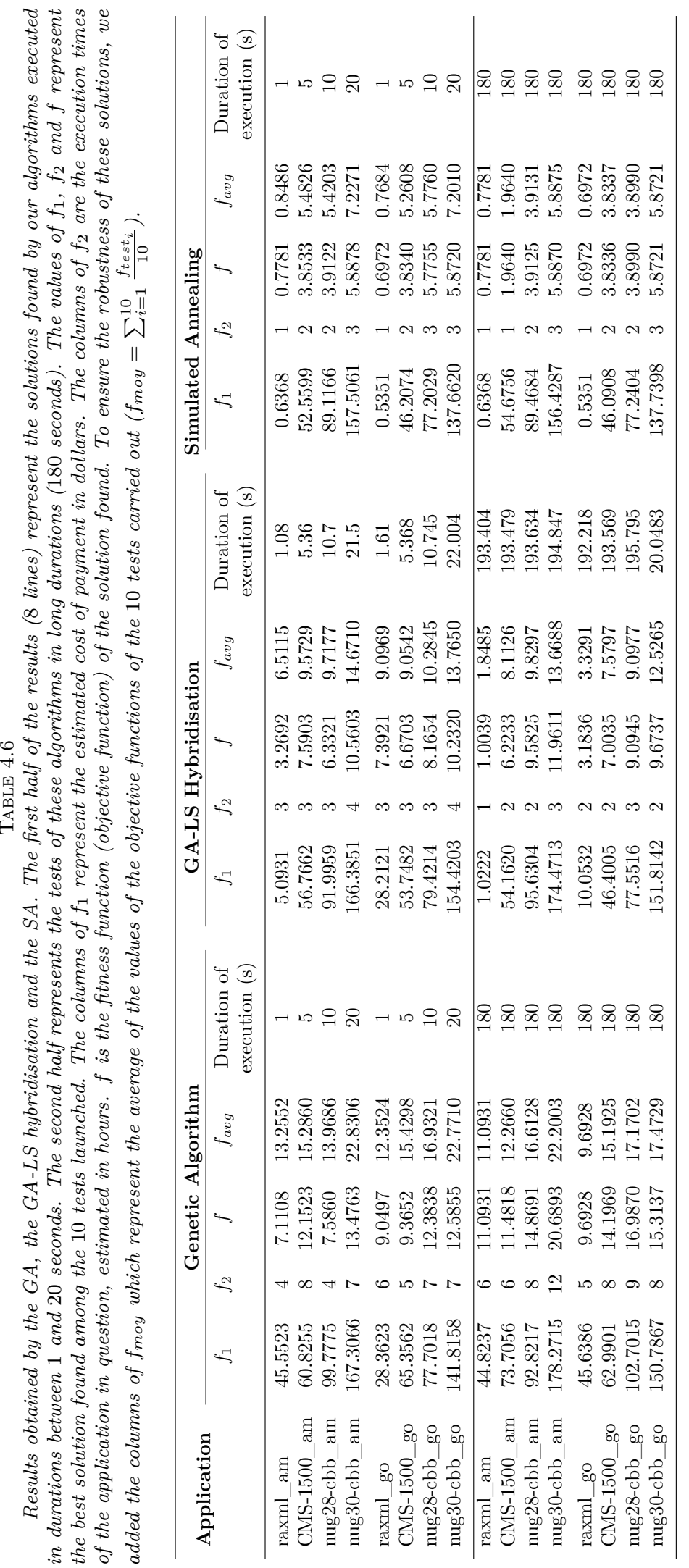




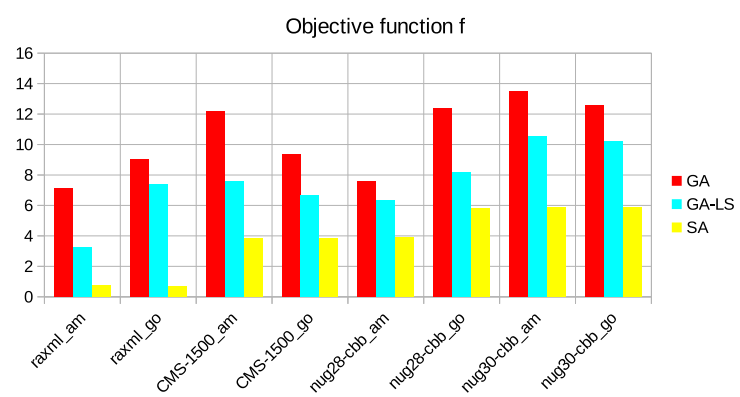

FIG. 4.1. Comparison of the objective function values found by the GA, the GA-LS hybridisation and the SA.

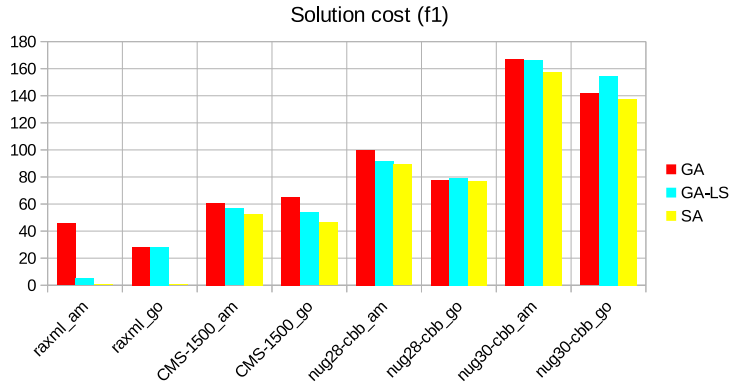

FIG. 4.2. Comparison of payment costs of solutions found by the GA, the GA-LS hybridisation and the SA.

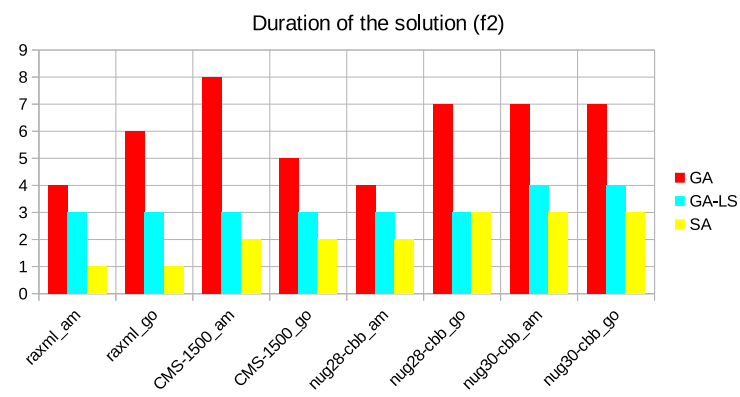

FIG. 4.3. Comparison of the durations of the solutions found by the GA, the GA-LS hybridisation and the SA.

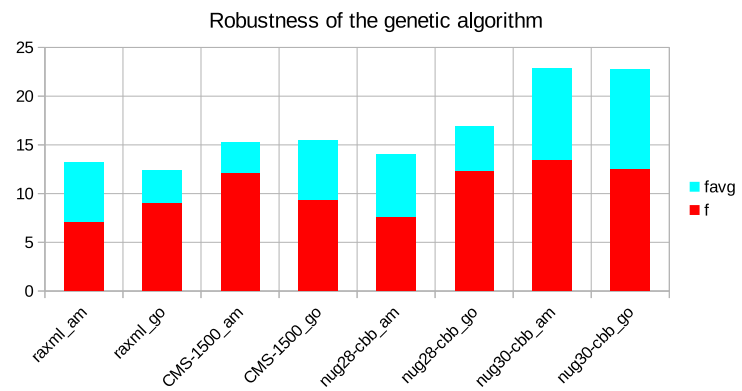

FIG. 4.4. Robustness of the GA verified by the comparison between the best solution found $(f)$ and the average of the solutions of the ten tests carried out $\left(f_{m o y}\right)$. 


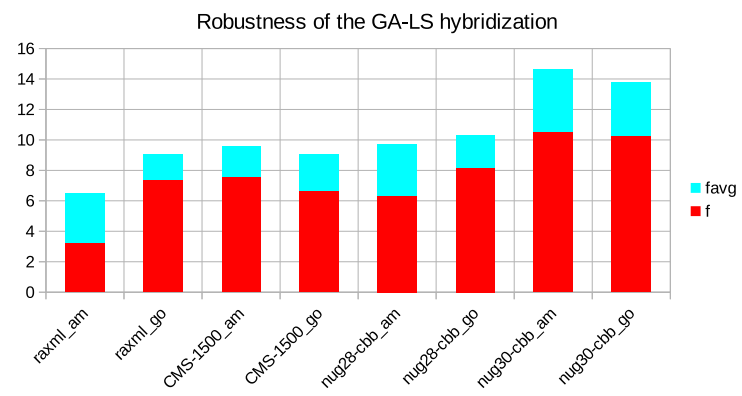

FIG. 4.5. Robustness of the GA-LS hybridisation verified by the comparison between the best solution found $(f)$ and the average of the solutions of the ten tests carried out $\left(f_{\text {avg }}\right)$.

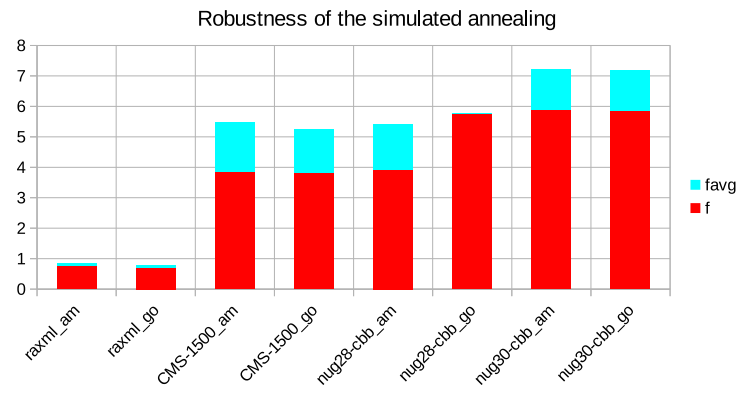

FIG. 4.6. Robustness of the $S A$ verified by the comparison between the best solution found $(f)$ and the average of the solutions of the ten tests carried out ( $\left.f_{\text {avg }}\right)$.

metaheuristics, namely the Genetic Algorithm, the hybridisation between the Genetic Algorithm and Local Search, and the Simulated Annealing. To compare between these approaches, we launched simulations on different sizes applications. The obtained results showed that the Simulated Annealing finds more optimal solutions than those proposed by the Genetic Algorithm and the hybridisation between the Genetic Algorithm and Local Search.

In the future, we plan to extend our work by comparing the Simulated Annealing with other metaheuristics, and test them in a different context such as the multi-cloud.

\section{REFERENCES}

[1] https://aws.amazon.com/fr/ec2/, Oct. 2017.

[2] https://cloud.google.com/compute/, Oct. 2017.

[3] https://azure.microsoft.com/fr-fr/, Feb. 2020.

[4] https://cloud.google.com/appengine/, Feb. 2020.

[5] https://gsuite.google.fr/intl/fr/, Feb. 2020.

[6] https://www.heroku.com/, Feb. 2020.

[7] https://www.office.com/, Feb. 2020.

[8] C. Blum and A. Roli, Metaheuristics in combinatorial optimization: Overview and conceptual comparison, ACM computing surveys (CSUR), 35 (2003), pp. 268-308.

[9] C. Collaboration, S. Chatrchyan, G. Hmayakyan, V. Khachatryan, A. Sirunyan, W. Adam, T. Bauer, T. Bergauer, H. Bergauer, M. Dragicevic, Et Al., The cms experiment at the cern lhc, 2008.

[10] R. D. C. Coutinho, L. M. Drummond, and Y. Frota, Optimization of a cloud resource management problem from a consumer perspective, in European Conference on Parallel Processing, Springer, 2013, pp. 218-227.

[11] J. Dréo, A. PÉtrowski, P. Siarry, and E. Taillard, Métaheuristiques pour l'optimisation difficile, 2003.

[12] M. Gabli, E. M. JaAra, And E. B. Mermri, A genetic algorithm approach for an equitable treatment of objective functions in multi-objective optimization problems., IAENG International Journal of Computer Science, 41 (2014)

[13] F. Glover and M. Laguna, Tabu search kluwer academic, Boston, Texas, (1997). 
[14] D. GoldBerg, Genetic algorithms in search, optimization, and machine learning, addison-wesley, reading, ma, 1989, NN Schraudolph and J, 3 (1989).

[15] A. D. Goncalves, L. M. Drummond, A. A. Pessoa, and P. Hahn, Improving lower bounds for the quadratic assignment problem by applying a distributed dual ascent algorithm, arXiv preprint arXiv:1304.0267, (2013).

[16] J. H. Holland, Outline for a logical theory of adaptive systems, Journal of the ACM (JACM), 9 (1962), pp. $297-314$.

[17] H. H. Hoos AND T. StützLe, Stochastic local search: Foundations and applications, Elsevier, 2004.

[18] S. Kirkpatrick, C. D. Gelatt, and M. P. Vecchi, Optimization by simulated annealing, science, 220 (1983), pp. 671-680.

[19] N. Metropolis, A. W. Rosenbluth, M. N. Rosenbluth, A. H. Teller, and E. Teller, Equation of state calculations by fast computing machines, The journal of chemical physics, 21 (1953), pp. 1087-1092.

[20] A. Mokhtari, M. AzIZI, AND M. Gabli, Optimizing management of cloud resources towards best performance for applications execution, in 2017 First International Conference on Embedded \& Distributed Systems (EDiS), IEEE, 2017, pp. 1-5.

[21] - Multi-cloud resources optimization for users applications execution, in International Conference Europe Middle East \& North Africa Information Systems and Technologies to Support Learning, Springer, 2018, pp. 588-593.

$[22] —$ - A fuzzy dynamic approach to manage optimally the cloud resources, in International Conference on Innovative Research in Applied Science, Engineering and Technology, IEEE, 2020

[23] J. Silberholz and B. Golden, Comparison of metaheuristics, in Handbook of metaheuristics, Springer, 2010, pp. 625-640.

[24] A. Stamatakis, Raxml-vi-hpc: maximum likelihood-based phylogenetic analyses with thousands of taxa and mixed models, Bioinformatics, 22 (2006), pp. 2688-2690.

[25] S.H.H. Madni, M.S.A. Latiff, Y. Coulibaly and S.I.M. Abdulhamid, An appraisal of meta-heuristic resource allocation techniques for IaaS cloud, Indian Journal of Science and Technology, 2016, 9(4), 1-14.

[26] U.K. Jena, P.K. DAS And M.R.Kabat, Hybridization of meta-heuristic algorithm for load balancing in cloud computing environment, Journal of King Saud University-Computer and Information Sciences, 2020.

Edited by: Dana Petcu

Received: Aug 14, 2020

Accepted: Dec 6, 2020 